\title{
Identificação dos Primeiros Sintomas do Autismo pelos Pais ${ }^{1}$
}

\author{
Regina Basso Zanon ${ }^{2}$ \\ Bárbara Backes \\ Cleonice Alves Bosa \\ Universidade Federal do Rio Grande do Sul
}

\begin{abstract}
RESUMO - Dificuldades no desenvolvimento social são os indicadores mais prováveis de um futuro diagnóstico de autismo, entretanto o atraso da fala parece ser o motivo que mais mobiliza os pais na busca por assistência. Neste estudo foram investigados os primeiros sintomas percebidos pelos pais de crianças com autismo e a idade da criança na ocasião. Participaram 32 pré-escolares e o instrumento utilizado foi a Autism Diagnostic Interview-Revised. Comprometimentos no desenvolvimento da linguagem foram os sintomas mais frequentemente observados, porém os da socialização foram os mais precocemente identificados. No geral, a idade média em que os primeiros sintomas foram percebidos foi 15,2 meses. Os resultados corroboram achados de outros estudos, ressaltando a importância dos comprometimentos sociais para a identificação precoce do autismo.
\end{abstract}

Palavras-chave: autismo, identificação precoce, percepção dos pais

\section{Parents' Perception of the First Symptoms of Autism}

\begin{abstract}
The occurrence of problems related to social development is the best predictors of a future diagnosis of an Autistic Disorder. Speech delay, however, seems to be the main that reason that leads parents to seek assistance. The present study aimed to investigate the first symptoms observed by the parents of children with autism and the age that it occurred. Thirty-two preschool children participated in the study. The instrument used was the Autism Diagnostic Interviwed-Revised. Abnormalities in language development were the most frequent symptoms reported by the parents, but social impairment was the earliest that was noticed. The average age when the parents noticed the first symptoms was 15.2 months. The results corroborate other findings, highlighting the importance of social deficits for the early identification of autism.
\end{abstract}

Keywords: autism, early identification, parental perception

Nos últimos anos, o termo Transtorno do Espectro do Autismo (TEA) vem sendo utilizado, nas publicações, para se referir a uma classe de condições neurodesenvolvimentais que, geralmente, inclui o transtorno autístico, o de Asperger, o desintegrativo da infância e o transtorno global do desenvolvimento não especificado, também conhecido como autismo atípico (Barbaro, 2009; Yoder, Stone, Walden, \& Malesa, 2009). Reconhecendo a natureza dimensional desse conjunto de condições que fazem parte do espectro e as controvérsias em relação ao diagnóstico diferencial entre elas, a quinta edição do Manual Diagnóstico e Estatístico de Transtornos Mentais (DSM-5) (American Psychiatric Association - APA, 2013), recentemente lançado e ainda não traduzido para o português, propõe a classificação de TEA em substituição a de Transtornos Globais do Desenvolvimento, adotada no DSM-IV-TR (APA, 2003). De acordo com a nova versão

1 Apoio: CAPES/Fulbright. Agradecimentos: À CAPES/Fulbrigth pelo apoio concedido para a realização da pesquisa. À Patricia Manning-Courtney, MD., professora associada do Departamento de Pediatria da Universidade de Cincinnati e diretora do Kelly O' Leary Center for Autism Spectrum Disorders do Cincinnati Children's Hospital Medical Center, pela orientação do projeto geral. À Dr Cynthia Molloy, MD pelos esclarecimentos concernentes ao banco de dados.

2 Endereço para correspondência: Universidade Federal do Rio Grande do Sul, Rua Ramiro Barcelos, 2600. Porto Alegre, RS. CEP 90035003. E-mail: rebzanon@gmail.com do manual, as manifestações comportamentais que definem o TEA incluem comprometimentos qualitativos no desenvolvimento sociocomunicativo, bem como a presença de comportamentos estereotipados e de um repertório restrito de interesses e atividades, sendo que os sintomas nessas áreas, quando tomados conjuntamente, devem limitar ou dificultar o funcionamento diário do indivíduo (APA, 2013).

O TEA é uma condição que tem início precoce e cujas dificuldades tendem a comprometer o desenvolvimento do indivíduo, ao longo de sua vida, ocorrendo uma grande variabilidade na intensidade e forma de expressão da sintomatologia, nas áreas que definem o seu diagnóstico. Atualmente, o TEA é compreendido como uma síndrome comportamental complexa que possui etiologias múltiplas, combinando fatores genéticos e ambientais (Rutter, 2011). Até o momento, as bases biológicas que buscam explicar a complexidade do transtorno são apenas parcialmente conhecidas e, por isso, a identificação e o diagnóstico do transtorno baseiam-se nos comportamentos apresentados e na história do desenvolvimento de cada indivíduo (Barbaro, 2009; Daley, 2004).

De acordo com estudos recentes, a estimativa de prevalência do TEA é 62/10.000 (ver Elsabbagh et al., 2012, para uma revisão), com uma incidência quatro vezes maior em meninos do que em meninas (Fombonne, 2009). De modo geral, o número de pessoas diagnosticadas mundialmente 
com transtorno é crescente, o que não indica, necessariamente, o aumento da sua prevalência. Esse fato pode ser explicado pela expansão dos critérios diagnósticos, pelo incremento dos serviços de saúde relacionados ao transtorno e pela mudança na idade do diagnóstico, dentre outros fatores (Fombonne, 2009).

Conforme os critérios diagnósticos do DSM-5 (APA, 2013), as primeiras manifestações do TEA devem aparecer antes dos 36 meses de idade. Todavia, dados empíricos demonstram que a maioria das crianças apresenta problemas no desenvolvimento entre os 12 e 24 meses (Chakrabarti, 2009; Chawarska et al., 2007; Noterdaeme \& Hutzelmeyer-Nickels, 2010), sendo que alguns desvios qualitativos no desenvolvimento aparecem antes mesmo dos 12 meses (Maestro et al., 2002; Zwaigenbaum et al., 2005).

Diversos estudos destacam a intervenção precoce como fator fundamental para a melhora do quadro clínico do autismo, gerando ganhos significativos e duradouros no desenvolvimento da criança (Howlin, Magiati \& Charman 2009; Reichow, 2011). Devido à plasticidade cerebral, a precocidade do início da intervenção desempenha papel importante, potencializando os efeitos positivos da mesma. Ademais, estudos indicam que os ganhos decorrentes da intervenção precoce podem reduzir consideravelmente os gastos dos familiares no tratamento das crianças com TEA, bem como os dos sistemas de saúde pública, quando se analisa os resultados em longo prazo (Järbrink \& Knapp, 2001; Mandell, Novak \& Zubristsky 2005).

Inúmeros aspectos podem retardar a intervenção, como é o caso da demora na detecção das primeiras dificuldades no comportamento da criança, na busca pela ajuda profissional e na realização do diagnóstico. De fato, alguns estudos têm demonstrado que crianças com TEA dificilmente recebem esse diagnóstico antes dos 5 anos (Daley, 2004; Howlin \& Asgharian, 1999; Mandell, Listerud, Levy \& Pinto-Martin, 2002), sendo que algumas são diagnosticadas apenas quando atingem idade escolar (Noterdaeme \& Hutzelmeyer-Nickels, 2010; Yeargin-Allsopp et al., 2003). Além disso, disparidades na idade média da realização do diagnóstico foram observadas quando considerados os diferentes grupos raciais e étnicos. Mandell et al. (2002) demonstraram, por exemplo, que crianças brancas são diagnosticadas aos 6,3 anos enquanto que as afro americanas o são aos 7,9 anos, em média. Essas diferenças raciais e étnicas na idade da realização do diagnóstico podem estar relacionadas a fatores institucionais, como dificuldades no acesso das famílias aos serviços de saúde (Mandell et al., 2009).

Siklos e Kerns (2007) ressaltam quatro fatores que podem influenciar no atraso na realização do diagnóstico precoce: 1) a variabilidade na expressão dos sintomas do TEA; 2) as limitações da própria avaliação de pré-escolares, uma vez que essa população demanda instrumentos específicos e sensíveis aos comportamentos sociais mais sutis e próprios dessa faixa etária; 3) a falta de profissionais treinados/habilitados para reconhecer as manifestações precoces do transtorno; e 4) a escassez de serviços especializados.

Até o momento, uma importante limitação do diagnóstico precoce refere-se ao fato de que muitas das características comportamentais do TEA, tal como constam nos manuais de classificação e de critérios diagnósticos, baseiam-se principalmente em sintomas que são comuns em crianças e adultos, mas que dificilmente são vistos em bebês (Gray \& Tonge, 2001; Johnson, 2008), como por exemplo, a interação com os pares. A mesma limitação aparece quando se analisam dificuldades no desenvolvimento da linguagem em crianças que ainda não falam, uma vez que o reconhecimento dos comprometimentos na área não-verbal (e.g., comunicação através dos gestos e expressões faciais/posturais), ainda é um desafio (Bosa, 2009; Johnson, 2008). Além disso, os comportamentos e interesses repetitivos e/ou rituais, dificilmente se manifestam antes dos 18 meses e tendem a se tornar mais aparentes aproximadamente entre os 3 e 4 anos (Gray \& Tonge, 2001; Turner, 1999), período em que a identificação do referido transtorno também é menos ambígua. Em vista disso, geralmente o encaminhamento das crianças com suspeita de TEA só ocorre quando se percebe um atraso importante na "fala", ainda que desvios no desenvolvimento da sociabilidade possam ser observados mais cedo (Bosa, 2009; 2002b).

Devido à convivência diária, envolvendo diferentes contextos e ocasiões, reconhece-se que, na maioria das vezes, são os pais, e não os profissionais, os primeiros a suspeitarem de problemas no desenvolvimento da criança. Coonrod e Stone (2004) destacam que as preocupações iniciais dos cuidadores são, geralmente, acuradas e legítimas. Sendo assim, a realização de entrevista com os pais é uma importante fonte de informação quando se pretende realizar o diagnóstico ou pesquisas, envolvendo crianças com TEA(Lord, Storoschuk, Rutter \& Pickles, 1993), ainda que haja limitações nesse procedimento. Entre os obstáculos, destacam-se os vieses e o desconhecimento por parte dos pais dos aspectos do desenvolvimento que são esperados para uma determinada idade. Este último aspecto, por si só, aponta para a necessidade de estudos e de divulgação dos seus resultados, para além da comunidade científica.

\section{Os cuidadores e o reconhecimento dos primeiros sintomas do TEA}

Nas últimas duas décadas, estudos têm buscado identificar a idade do reconhecimento dos primeiros sintomas (IRPS) do TEA, através de entrevistas realizadas com os pais de crianças diagnosticadas com esse transtorno. Resultados mostram que os primeiros sintomas no desenvolvimento tendem a ser percebidos pelos pais durante os dois primeiros anos de vida, sendo que a IRPS varia de 14,7 meses $(D P=6,5)$ a 23,4 meses $(D P=11,3)$ (Chakrabarti, 2009; Chawarska et al., 2007).Não surpreendentemente, observou-se uma correlação positiva entre a idade da amostra e a IRPS, isto é, quanto menor a idade da criança, na época do estudo, mais cedo o reconhecimento dos primeiros sintomas pelos pais (Chakrabarti, 2009; Chawarskaet al., 2007; Coonrod \& Stone, 2004; De Giacomo \& Fombonne, 1998). A justificativa é que, no caso de crianças mais velhas, o intervalo entre as primeiras manifestações sintomáticas e a coleta de dados com os cuidadores pode gerar distorções nas informações, pois estas dependem da lembrança de detalhes do desenvolvimento da criança. 
Sobre a relação entre a IRPS e outros aspectos da vida da criança, tem sido demonstrado que a deficiência intelectual e problemas médicos associados, nas crianças, tendem a antecipar a IRPS pelos pais (De Giacomo \& Fombonne, 1998; Noterdaeme \& Hutzelmeyer-Nickels, 2010; Shattucket al., 2009; Wiggins, Baio, \& Rice, 2006). Da mesma forma, complicações perinatais e déficits sensoriais também levam os cuidadores a reconhecer os sintomas mais precocemente (Baghdadli, Picot, Pascal, Pry, \&Aussilloux, 2003). Por outro lado, a ordem de nascimento, a classe social e o gênero da criança são fatores que parecem não estar vinculados diretamente à IRPS (Chawarska et al., 2007; Daley, 2004; Noterdaeme \& Hutzelmeyer-Nickels, 2010).

Em relação à natureza dos primeiros sintomas observados pelos pais, o atraso no desenvolvimento da comunicação e da linguagem é o sintoma relatado com maior frequência (Chakrabarti, 2009; Chawarska et al., 2007; Coonrod et al., 2004; De Giacomo \& Fombonne, 1998; Howlin \& Asgharian, 1999). Por outro lado, pesquisas demonstram que os comprometimentos no desenvolvimento social são os primeiros sintomas a emergirem, embora reconhecidos apenas por uma pequena parcela dos pais (Johnson, 2008; Werner, Dawson, Munson, \& Osterling, 2005). Contudo, ressalta-se que os estudos retrospectivos raramente especificam quais aspectos das áreas comprometidas pelo transtorno os pais são capazes de identificar.

Preocupações iniciais concernentes a aspectos da brincadeira, do desenvolvimento motor, da alimentação e do sono também foram reportadas por cuidadores de crianças com TEA (Daley, 2004; Werner \& Dawson, 2005). Além disso, um estudo realizado com 36 crianças com TEA e 20 controles, com desenvolvimento típico, e seus pais, encontrou que de 20 a $30 \%$ dos cuidadores descrevem padrões de regressão de linguagem, envolvendo a perda de palavras previamente adquiridas (Werner \& Dawson, 2005). De fato, investigações recentes acerca do tema têm demonstrado que a regressão é um fenômeno fidedigno, que afeta uma parcela importante das crianças com TEA (Backes, Zanon, \& Bosa, 2013; Wiggins, Rice, \& Baio, 2009). Por exemplo, Backes et al. 2013; Backes, Zanon, Endres, Meimes, \& Bosa, 2012, em um estudo realizado com 30 crianças americanas com Transtorno Autista, conforme critérios diagnósticos da Autism Diagnostic Interview-Revised (ADI-R), encontraram que $20 \%$ delas apresentaram regressão das habilidades de linguagem. A média de idade de início da perda encontrada nessa pesquisa foi de 25 meses $(D P=6,19)$, o que se aproxima de achados de outras investigações (Pickles et al., 2009; Werner \& Dawson, 2005).

Werner et al. (2005) realizaram um estudo retrospectivo, analisando as variações no desenvolvimento social precoce (0-2 anos) de crianças e suas relações com as manifestações comportamentais aos 3-4 anos de vida. Participaram desse estudo 145 crianças americanas, sendo 39 com desenvolvimento típico, $34 \mathrm{com}$ atraso no desenvolvimento e $72 \mathrm{com}$ TEA. Os grupos foram equiparados em termos de desempenho cognitivo, nível socioeconômico e etnia. Para investigar o desenvolvimento precoce das crianças, foi utilizada a Early Development Interview, uma entrevista composta por 96 itens, que foram desenvolvidos com o intuito de aumentar a qualidade da memória dos pais. Perceberam-se diferenças significativas entre o desenvolvimento social das crianças com TEA e das crianças com desenvolvimento típico, já no segundo trimestre de vida. No entanto, quando comparadas às crianças com atraso no desenvolvimento, as diferenças se acentuaram somente após os 13-15 meses. Nessa idade, as crianças com TEA apresentaram sintomas como pouco contato ocular, falta de orientação ao ser chamado pelo nome e de engajamento em interações sociais, sobretudo na habilidade de atenção compartilhada. Nessa pesquisa, os autores concluíram que os comportamentos sociais são os melhores indicadores para o diagnóstico diferencial entre crianças com TEA e aquelas com desenvolvimento típico ou com atraso no desenvolvimento. Tais achados são similares aos de outros estudos realizados com base na análise de vídeos domésticos (ver Saint-Georges et al., 2010, para uma revisão).

Ozonoff et al. (2010), através de um estudo prospectivo e longitudinal, que comparou o desenvolvimento de bebês posteriormente diagnosticados com TEA com o de bebês com desenvolvimento típico, encontraram que a frequência do olhar para faces, do sorriso social e das vocalizações só começou a declinar a partir dos seis meses de idade no grupo com TEA. Antes disso, os grupos eram altamente comparáveis, o que chama a atenção para o segundo semestre de vida como um período crítico na emergência de comprometimentos mais substanciais do espectro.

Cabe salientar que é justamente no segundo semestre de vida, mais precisamente ao redor dos 9 meses de idade do bebê, que emerge uma habilidade sociocomunicativa exclusivamente humana, da qual decorrem mudanças significativas na maneira como o bebê passa a se relacionar com os outros, com os objetos e consigo mesmo (Tomasello, 1999/2003). A habilidade de compartilhar as descobertas sobre o mundo (atenção compartilhada), através do olhar, da atividade gestual (e.g., apontar, mostrar e dar objetos para os outros) e de expressões emocionais, é um marco no desenvolvimento sociocomunicativo da criança, cuja ausência é um importante elemento diagnóstico do TEA. De fato, estudos demonstram que o comprometimento e o atraso no desenvolvimento da habilidade de atenção compartilhada é um dos sinais mais precocemente observados em crianças com TEA (Bosa, 2009, 2002a; Naber et al., 2008; Shumway \& Wetherby, 2009), sendo capaz de discriminar de 80 a $90 \%$ de crianças com o transtorno de outras com outros problemas ou atrasos no desenvolvimento (Charman et al., 1997).

Os aspectos apresentados até agora apontam para a importância da investigação e difusão dos marcadores pré-linguísticos do TEA, em diferentes centros de excelência no diagnóstico do transtorno. Esses achados podem subsidiar o processo de construção de instrumentos que podem ser administrados em diferentes contextos (e.g., médico, educacional - berçário). Em vista dessa importância, o presente estudo tem como objetivo investigar as dificuldades específicas do espectro do autismo percebidas pelos pais, nos primeiros anos de vida do filho, bem como a idade da criança na ocasião. 


\section{Método}

\section{Delineamento e Participantes}

Trata-se de um estudo de cunho retrospectivo e descritivo (Robson, 1995), que utilizou um banco de dados internacio$\mathrm{nal}^{3}$, tendo em vista a necessidade de se realizar a pesquisa com base em instrumentos diagnósticos fidedignos, e ainda não completamente validados no Brasil.

Participaram 32 crianças em idade pré-escolar, que foram atendidas no Cincinnati Children's Hospital Medical Center (CCHMC), em Ohio (EUA), nos anos 2008 e 2009. Todas as crianças são caucasianas e têm o diagnóstico de Transtorno Autista, realizado por especialistas, utilizando entre outros instrumentos, a ADI-R (Lord et al., 1993). A média de idade das crianças é 4,17 anos $(D P=1,06)$ e a maioria delas é do sexo masculino $(75,75 \%)$, com uma proporção em relação ao sexo feminino de 5,4:1.

\section{Instrumentos}

Neste estudo, a IRPS por parte dos cuidadores e a natureza dos mesmos basearam-se na parte introdutória da ADI-R, mais precisamente na segunda sessão da entrevista, na qual é investigado o desenvolvimento precoce da criança. A ADI-R é uma entrevista padrão ouro, semiestruturada e administrada aos cuidadores. É requerido ao entrevistador um treinamento prévio para a administração do instrumento e para a codificação dos resultados, no qual ele é instruído a obter descrições detalhadas dos pais acerca do comportamento do filho, buscando através de cada pergunta exemplos claros dos comportamentos investigados (Lord et al., 1993). Foram considerados neste estudo os seguintes itens da ADI-R: a) item 2 - idade, em meses, quando os pais notaram pela primeira vez que algo não estava bem nas áreas da linguagem, do relacionamento social ou do comportamento; b) item 3 - primeiros sintomas que causaram preocupações aos pais; e c) item 4 - percepção inicial, em retrospecto. Para fins de análise, o presente estudo contou com as respostas dos cuidadores a cada um dos itens, que foram registradas na íntegra no banco de dados, sendo submetidas, posteriormente, à análise de conteúdo quantitativa (Bardin, 1977). Em relação aos aspectos éticos envolvidos na pesquisa, ressalta-se que houve a autorização formal para o uso do banco de dados, sendo a identidade dos participantes mantida em sigilo, no próprio banco de dados repassado aos pesquisadores.

\section{Resultados}

Quatro categorias foram criadas para a análise da natureza dos primeiros sintomas observados, quais sejam: 1) atraso/peculiaridades no desenvolvimento da linguagem; 2) problemas

3 O presente estudo faz parte de um projeto conjunto entre o Programa de Pós-Graduação de Psicologia da Universidade Federal do Rio Grande do Sul e o Centro de Transtornos do Desenvolvimento do Cincinnati Children's Hospital Medical Center, financiado pela CAPES/Fulbright. no comportamento social; 3) comportamento estereotipado e repetitivo; 4) atraso/peculiaridade no desenvolvimento de outras áreas do desenvolvimento. Nos casos em que os informantes relataram preocupações em relação a mais de um comportamento da criança, estes foram classificados em mais de uma categoria (i.e., respostas múltiplas). Por essa razão, foram contabilizadas um total de 49 respostas/ comportamentos.

Evidenciou-se que $83,67 \%$ dos comportamentos relatados $(n=41)$ corresponderam a uma das três áreas comprometidas pelo TEA: atraso/peculiaridade no desenvolvimento da linguagem $(36,73 \%, n=18)$, problemas no comportamento social $(30,61 \%, n=15)$ e comportamento estereotipado e repetitivo $(16,33 \%, n=8)$. Os demais sintomas observados $(16,33 \%, n=8)$ referiram-se a outras áreas do desenvolvimento, incluindo problemas no sono, na alimentação e no desenvolvimento motor.

No que se refere especificamente ao desenvolvimento da linguagem, quatro subcategorias foram criadas: 1) atraso na fala (e.g., speech not coming [a fala não vinha]); 2) atraso na comunicação gestual (e.g., no waving [não abanava]; (3 atraso no balbucio (e.g., when baby not babbling [quando bebê não balbuciava]); e 4) "parou" de falar (e.g., stopped talking [parou de falar]). Evidenciou-se que o atraso na fala foi o sintoma mais relatado pelos pais $(61,1 \%, n=11)$, seguido pelo atraso na comunicação gestual $(16,7 \%, n=3)$, pelo atraso no balbucio $(11,1 \%, n=2)$ e pelo fato de a criança ter "parado" de falar $(11,1 \%, n=2)$ (Figura 1).

No que tange aos comprometimentos observados no desenvolvimento social, os comportamentos foram analisados de acordo com as seguintes subcategorias: 1) interação (e.g., freaked out in small groups [ficava apavorado quando em pequenos grupos]); 2) olhar/sorriso (e.g., when baby wouldn't look [quando bebê, não olhava]); 3) não responde ao ser chamado pelo nome (e.g., not responding to name [não respondia ao nome]); e 4) não responde à separação dos pais (e.g., nevercared if parents left [não se importava quando os

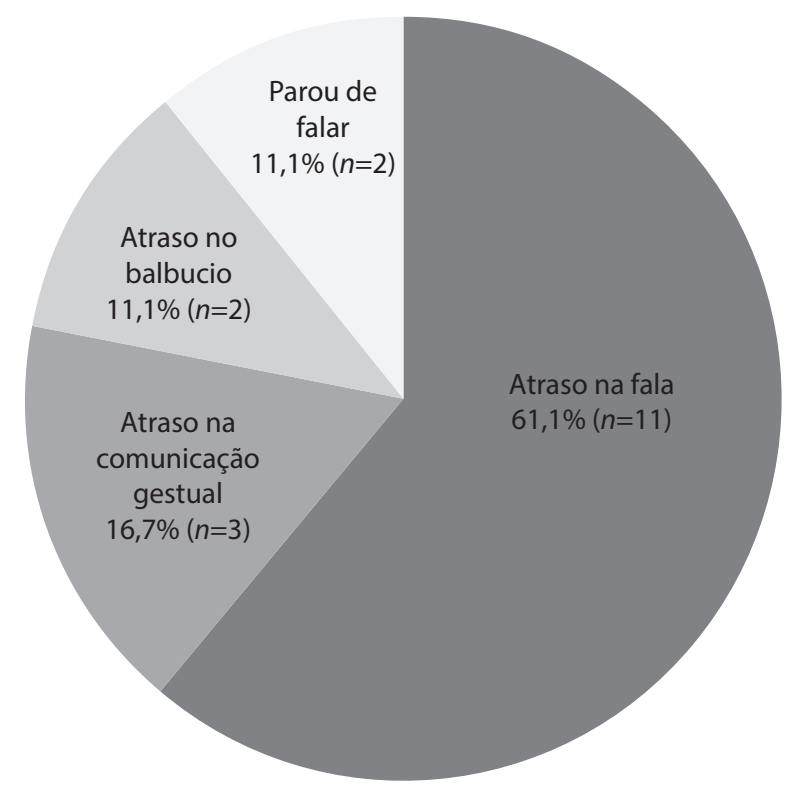

Figura 1. Frequência dos primeiros sintomas no desenvolvimento da linguagem. 


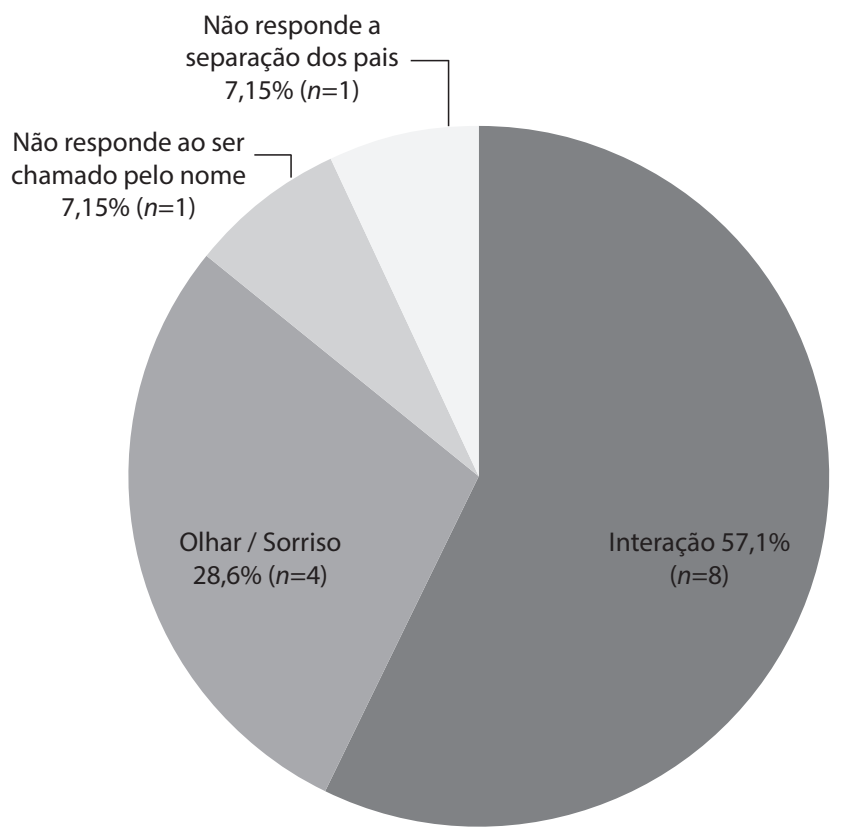

Figura 2. Frequência dos primeiros sintomas observados no desenvolvimento social.

pais o deixavam]). Encontrou-se que os problemas na interação, incluindo ansiedade, aversão, medo e/ou indiferença no contato com outras pessoas, foram os comportamentos mais identificados pelos pais, representando $57,1 \%(n=8)$ do total nessa subcategoria, seguidos pelos problemas na qualidade do olhar/sorriso $(28,6 \%, n=4)$, na ausência de resposta da criança ao ser chamado pelo nome $(7,15 \%, n=1)$ e pelo fato de ela não responder à separação dos pais $(7,15 \%, n=1)$ (Figura 2).

Por fim, no que se refere aos comportamentos repetitivos e estereotipados, os relatos foram classificados, de acordo com as seguintes subcategorias: 1) brincadeira repetitiva (e.g., spinning wheels as soon as he could [girava rodas o mais rápido que conseguia]); 2) rituais e interesses circunscritos (e.g., memorizing books [memorizava livros]); e 3) maneirismos e estereotipias (e.g., started stimming [começou a apresentar maneirismos]). Verificou-se que preocupações precoces quanto à qualidade da brincadeira dos filhos, incluindo ações de girar e/ou classificar objetos, representaram $50 \%(n=4)$ dos comportamentos relatados pelos pais. Foram destacadas também, nessa categoria, preocupações concernentes aos rituais e interesses circunscritos $(25 \%, n=2)$ e aos maneirismos e estereotipias $(25 \%, n=2)$ (Figura 3).

Em relação ao aspecto idade, a média de idade das crianças quando os pais observaram os primeiros sintomas no desenvolvimento foi 16,43 meses $(D P=11,39)$. Por outro lado, quando consideradas somente as manifestações precoces nas três áreas características dos TEA, a média da IRPS caiu para 15,17 meses $(D P=8,58)$. Os sintomas na área da socialização foram os mais precocemente observados pelos pais, durante o segundo semestre de vida da criança $(M=9,27$ meses; $D P=7,01)$. As alterações no desenvolvimento da linguagem foram os segundos sinais a serem identificados $(M=18,28$ meses; $D P=8,28)$, seguidos pelos comportamentos estereotipados e repetitivos $(M=19,25$ meses; $D P=6,11)$, ambos percebidos entre o primeiro e o segundo ano de vida da criança. A média de idade das crianças cujos pais

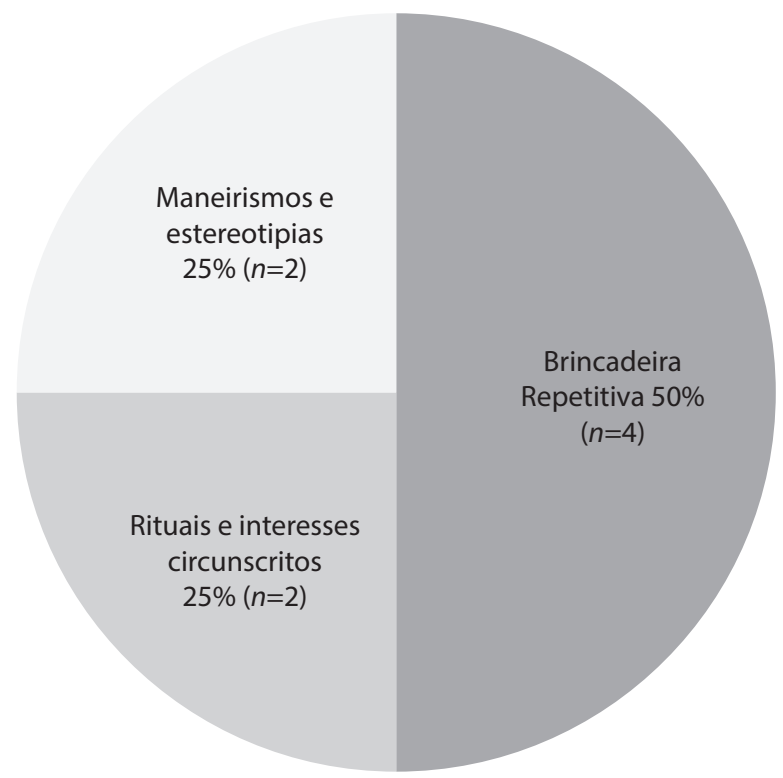

Figura 3. Frequência dos primeiros sintomas observados no desenvolvimento dos comportamentos estereotipados e repetitivos.

identificaram atraso/peculiaridade em outras áreas do desenvolvimento, que não as que caracterizam o TEA, foi 22,87 meses $(D P=20,29)$.

A maioria dos pais $(43,75 \%, n=14)$ afirmou ter reconhecido os primeiros problemas entre o primeiro e o segundo ano de vida do filho, enquanto que $28,12 \%$ deles $(n=9)$ identificaram antes do primeiro aniversário e, do mesmo modo, 28,12\% $(n=9)$, após o segundo ano da criança. Entretanto, quando, em outro momento da entrevista, foi novamente perguntado aos pais qual o período mais precoce do desenvolvimento no qual algo "anormal" possa ter ocorrido, as respostas dos cuidadores indicaram que quase a metade das crianças (43,75\%; $n=14$ ) apresentou problemas nos primeiros 12 meses de vida; $43,75 \%(n=14)$ entre o primeiro e o segundo ano de vida; e $12,5 \%(n=4)$ com dois anos ou mais.

\section{Discussão}

Os dados obtidos no presente estudo demonstram que os pais das crianças com autismo foram capazes de perceber dificuldades no desenvolvimento do filho ainda antes do segundo ano de vida da criança, quando consideradas apenas as manifestações específicas do espectro, corroborando outros achados (Chakrabarti, 2009; Chawarska et al., 2007; Noterdaeme \& Hutzelmeyer-Nickels, 2010). Além disso, estudos prospectivos realizados com irmãos de indivíduos com TEA, cujo risco estimado de também apresentarem o transtorno é de 5 a 10\% (Rice et al., 2007), têm identificado diversos comportamentos manifestados entre 12 e 18 meses, que distinguem os bebês com TEA de outros com desenvolvimento típico (Zwaigenbaum et al., 2009).

Quanto à natureza dos primeiros sintomas observados, verificou-se que, consistentes com outros estudos retrospectivos, a maioria das preocupações relatadas referiu-se ao desenvolvimento da linguagem, sobretudo em relação 
ao desenvolvimento da fala, seguido pelos problemas no comportamento social e nos comportamentos repetitivos e estereotipados (Chakrabarti, 2009; Chawarska et al., 2007; Coonrod \& Stone, 2004). Entretanto, investigações baseadas em vídeos domésticos ou pesquisas prospectivas mostram que os comprometimentos sociais são os sinais que mais frequentemente aparecem, sendo anteriores ao desenvolvimento da linguagem oral (Saint-Goerges et al., 2010; Ozonoff et al., 2010).

Os comprometimentos na área da linguagem, mais frequentemente identificados pelos pais, foram reconhecidos entre o primeiro e o segundo ano de vida da criança. $\mathrm{O}$ mesmo aconteceu com os comportamentos estereotipados e repetitivos, que também foram identificados nesse período. Watt, Wetherby, Barber e Morgan (2008) analisaram os comportamentos estereotipados e repetitivos, porém em 50 crianças com TEA, 25 com atraso no desenvolvimento e 50 com desenvolvimento típico, entre os 18 e 24 meses de idade. Os pesquisadores encontraram que as crianças com TEA apresentaram os comportamentos investigados com maior frequência e duração que as crianças dos outros grupos, envolvendo movimentos estereotipados e repetitivos com o próprio corpo, com os objetos, além de comportamentos sensoriais. Por tal razão, entende-se que os comportamentos repetitivos e estereotipados também são importantes para a identificação precoce do TEA.

De modo geral, no presente estudo, os problemas no desenvolvimento social da criança foram os mais precocemente identificados pelos pais, ainda no segundo semestre de vida do filho. A maioria dos pais que reconheceu os primeiros sintomas nessa área relatou dificuldades da criança na interação social. Estes resultados corroboram achados de outros estudos sobre o tema (Jonhson, 2008; Werner et al., 2005), apontando para a importância dos comprometimentos sociais para a identificação precoce do TEA.

Evidenciou-se, também, que algumas dificuldades na interação social, (e.g., medo, ansiedade, aversão ou indiferença na relação com outras pessoas) foram identificadas pelos pais um pouco depois dos 9 meses de idade da criança. Ressalta-se que comportamentos dessa natureza, que comprometem a relação da criança com os outros, são distantes do tipicamente esperado para essa faixa etária. De fato, Papaeliou e Trevarthen (2006) observaram que bebês com desenvolvimento típico, aos 10 meses, preferem brincadeiras de engajamento coordenado com pares ao invés de brincadeiras solitárias. Além disso, vale dizer que esse período representa um avanço substancial no desenvolvimento dos processos sociocomunicativos, devido ao surgimento da habilidade de Atenção Compartilhada (AC), que se desenvolve em contextos de trocas sociais (Tomasello, 1999/2003). Nesse sentido, pode-se pensar que uma criança que apresenta, no final do primeiro ano de vida, aversão, ansiedade ou medo do contato com outras pessoas provavelmente apresentará déficits na $\mathrm{AC}$ e, consequentemente, comprometimentos no desenvolvimento da linguagem oral, aspecto mais frequentemente relatado pelos pais. Isso ocorre porque, segundo as premissas das teorias interacionistas, a AC é a base para o desenvolvimento social e linguístico subsequente (Tomasello, 1999/2003).
Os resultados desta pesquisa mostraram ainda que, em uma análise retrospectiva, com base na ADI-R, os mesmos pais passaram a reconhecer manifestações do TEA ainda mais precoces do que aquelas que inicialmente geraram preocupações. Ou seja, os cuidadores identificaram dificuldades anteriores aos comprometimentos observados entre o primeiro e segundo ano de vida da criança, que se referiram, principalmente, ao atraso no desenvolvimento da linguagem oral. As mudanças evidenciadas no julgamento dos pais, quanto ao momento em que os primeiros sintomas apareceram, podem ter sido efeito do próprio processo de uma avaliação diagnóstica detalhada, realizada em um centro especializado e com instrumentos reconhecidos. Nesse sentido, pensa-se que o fato de já ter iniciado um processo de avaliação profissional acerca dos comportamentos apresentados pela criança, pode ter atentado os pais para aspectos próprios do desenvolvimento infantil precoce, bem como para as manifestações comportamentais do TEA, que são sutis durante os primeiros anos de vida.

Nessas circunstâncias, depreende-se do presente estudo que há uma sincronia desenvolvimental importante entre as interações sociais iniciais e o desenvolvimento sóciocomunicativo posterior. Com isso, pensa-se, inclusive, que o atraso na fala, observado por grande parte dos pais entre o primeiro e o segundo ano da criança, ainda na etapa das holófrases (i.e., quando uma palavra, usada sozinha, é capaz de corresponder a uma frase) (Tomasello, 1999/2003), pode ter decorrido de comprometimentos mais precoces na interação social que, por se tratarem de comportamentos sutis, podem não ter sido identificados pelos pais no momento em que surgiram. No entanto, pode-se supor que tais manifestações sutis, concernentes à interação social, passaram e ser reconhecidas por um número maior de pais no momento da entrevista, mostrando um possível efeito positivo do processo de avaliação.

Assim, destacam-se algumas implicações dos achados do presente estudo. Embora pesquisas recentes tenham demonstrado a existência de comprometimentos precoces no desenvolvimento sociocomunicativo, em especial na habilidade de $\mathrm{AC}$, os profissionais da saúde devem reconhecer que a maioria dos pais das crianças com TEA não relatam, espontaneamente, dificuldades precoces nessa área. Adrien et al. (1992), em um estudo baseado em vídeos caseiros, afirmam, inclusive, que os déficits sociais estão presentes na criança em uma idade anterior àquela que primeiro preocupou os seus pais. Tal fato pode ser reflexo da falta de informação dos pais acerca dos marcos do desenvolvimento social (e.g., gestos e outros comportamentos envolvidos na AC), especialmente quando em comparação com os do desenvolvimento da linguagem. Outra explicação possível para o não reconhecimento dos sinais no desenvolvimento social consiste no fato de alguns pais atribuírem a falta de interação da criança a aspectos da personalidade do filho, independentes do TEA (Sifuentes \& Bosa, 2007), como a timidez ou introversão ou, ainda, a aspectos do ambiente, como pouca estimulação, muito "mimo", dentre outros fatores.

Por tais razões, alerta-se que a falta dos relatos parentais acerca dos comprometimentos sociais são significa, necessariamente, a ausência dessas dificuldades. Recomenda-se, assim, que os profissionais indaguem os cuidadores espe- 
cificamente acerca do desenvolvimento social, buscando detalhes e exemplos claros dos mesmos, sobretudo quando a queixa principal consiste apenas no atraso no desenvolvimento da linguagem oral. Tais cuidados são importantes porque o reconhecimento dos sinais do TEA, ainda na primeira infância, constitui um primeiro passo na direção da realização do diagnóstico precoce, fato que aumenta a possibilidade de a criança se beneficiar dos efeitos da intervenção.

Especificamente no que se refere ao contexto brasileiro, ressalta-se a escassez de instrumentos validados para rastreamento e diagnóstico do TEA, um importante limitador para os profissionais e os pesquisadores na área. Por tal razão, recomenda-se a realização de pesquisas em nosso país que tenham como objetivo construir e validar instrumentos específicos para esse transtorno, em especial aqueles voltados para crianças bem pequenas. Nessa linha, ressalta-se a relevância de algumas dissertações realizadas nos últimos cinco anos por autores brasileiros, como, por exemplo, a de Montenegro e Mercadante (2007), na qual foi desenvolvido o Protocolo de Avaliação da Comunicação Social Inicial, um método de observação estruturada da orientação social e da AC. Ainda se tratando de instrumentos baseados na observação direta da criança, Marques e Bosa (2010) apresentaram evidências de validade preliminares do Protocolo de Observação para Crianças com Suspeita de TEA, que leva em conta a frequência, a intensidade e a peculiaridade dos sintomas próprios dos TEA, avaliados durante cenas de interação livres e estruturadas com um adulto. Destaca-se, também, o estudo desenvolvido por Castro-Souza e Pasquali (2011), que realizaram a adaptação brasileira do Modified Checklist for Autism in Toddlers, um instrumento de triagem precoce de TEA, composto por 23 itens dicotômicos do tipo sim/não, que foi projetado para ser respondido por pais ou responsáveis de crianças na faixa de idade de 18 a 24 meses. Além disso, foi realizada recentemente a tradução, bem como as análises preliminares de evidências de validade,da ADI-R para o português brasileiro (Becker et al., 2012), o que representa um campo promissor para a replicação do presente estudo em nosso país.

A presente pesquisa apresentou algumas limitações, que merecem ser consideradas. Muitas delas referem-se ao fato de ter sido utilizado parte de um banco de dados internacional. A consequência foi a falta de informações importantes sobre o nível de escolaridade parental, bem como acerca do desenvolvimento cognitivo das crianças, aspectos esses que podem influenciar na IRPS (Noterdaeme \& Hutzelmeyer-Nickels, 2010; Shattuck et al., 2009). Do mesmo modo, ressalta-se que o tamanho da amostra foi reduzido substancialmente devido, principalmente, ao fato de muitas das crianças que passaram pelo Cincinnati Children's Medical Center, especificamente em 2008 e 2009, não terem sido avaliadas pela ADI-R. A falta de grupo controle e/ou comparativo também nos impede de afirmar que os comportamentos relatados são específicos do grupo analisado.

Outra limitação refere-se ao instrumento utilizado. Pelo fato de a ADI-R se tratar de uma entrevista, as informações acerca dos primeiros sintomas apresentados pela criança são investigadas retrospectivamente. Por isso, deve-se considerar o efeito do tempo na memória dos entrevistados, fenômeno denominado, na literatura internacional, de "telescópico" [telescopingeffect] (Hus, Taylor, \& Lord, 2011; Sudman \& Bradburn, 1973). Esse fenômeno, comumente discutido em estudos retrospectivos, considera alguns fatores que podem influenciar a qualidade e a precisão da informação coletada: 1) intervalo de tempo entre o evento investigado e a coleta de dados; 2) qualidade da resposta dada pelo informante; e 3) estado emocional do informante (Hus et al., 2011). No que se refere ao presente estudo, vale considerar que a idade da amostra (pré-escolares), bem como a qualidade reconhecida do instrumento utilizado (padrão-ouro para avaliação do TEA) podem ter reduzido os efeitos do fenômeno telescópico, embora não se possa afirmar a não influência do mesmo. Ressalta-se que fazem parte do treinamento dos entrevistadores técnicas de entrevista no sentido de reduzir esse efeito.

\section{Considerações finais}

Com o presente estudo, pode-se concluir que os pais, pelo menos na cultura estudada, foram capazes de reconhecer sintomas próprios do TEA durante os dois primeiros anos de vida do filho. Esse aspecto é fundamental porque é um gatilho na busca por auxílio médico, em uma cadeia de acontecimentos que pode culminar com o diagnóstico precoce. Os comprometimentos no comportamento social foram os mais precocemente observados pelos cuidadores, sobretudo a qualidade da interação social. Uma vez que habilidades sóciocomunicativas básicas, como é o caso da AC, desenvolvem-se na relação com outras pessoas, presumiu-se, neste estudo, que problemas precoces na interação podem ter acarretado atraso na fala, comportamento relatado com maior frequência pelos pais. Tal fato ressalta a importância da realização de um rastreamento detalhado acerca do desenvolvimento sócio comunicativo precoce da criança, mesmo que os cuidadores não tenham relatado preocupações iniciais nessa área. Isso porque a detecção de problemas no desenvolvimento social, em especial na AC, é crucial para o diagnóstico do TEA, maximizando a probabilidade de a criança receber intervenção precoce em serviços especializados.

Do ponto de vista dos desdobramentos deste estudo, sugere-se que pesquisas futuras sejam realizadas a fim de melhor esclarecer algumas questões, como por exemplo, o papel da escolaridade parental e do desenvolvimento cognitivo da criança como um possível facilitador deste processo. Do mesmo modo, recomenda-se que pesquisadores e profissionais nessa área investiguem as primeiras manifestações sintomáticas, através de instrumentos específicos para essa faixa etária, a fim de possibilitar a recordação dos pais acerca dos sinais mais sutis. No que se refere ao contexto brasileiro, a questão é ainda anterior, uma vez que pesquisas envolvendo a construção e validação de instrumentos específicos para o TEA são muito iniciais, principalmente no que tange aos de rastreamento de casos suspeitos. A replicação do presente estudo com amostras brasileiras também se faz necessária. Além disso, estudos longitudinais e prospectivos são importantes para melhor investigar os indicadores precoces do TEA, sendo este conhecimento fundamental para se pensar em programas com vistas a habilitar pais e profissionais a identificarem precocemente esse transtorno. 


\section{Referências}

Adrien, J. L., Perrot, A., Sauvage, D., Leddet, I., Larmande, C., Hameury, L., \& Barthelemy, C. (1992). Early symptoms in autism from family home movies.Acta Paedopsychiatrica, 55(2), 71-75.

American Psychiatric Association (2013). Diagnostic and Statistical Manual of Mental Disorders (5a. ed.). Arlington, VA: American Psychiatric Publishing.

American Psychiatric Association. (2003). Manual diagnóstico e estatístico de transtornos mentais (4a. ed., Dornelles, C. trad.). Porto Alegre: Artmed.

Backes, B., Zanon, R. B., \& Bosa, C. A. (2013). A relação entre regressão da linguagem e desenvolvimento sociocomunicativo de crianças com transtorno do espectro do autismo. CoDAS, 25(3), 268-273.

Backes, B., Zanon, R. B., Endres, R. G., Meimes, M. A., \& Bosa, C. A. (2012, May). The regression of language skills in preschool children with Autism Spectrum Disorder. Postersession presented at the International Meeting for Autism Research, Toronto, Canada.

Baghdadli, A., Picot, M. C., Prascal, C., Pry, R., \& Aussilloux, C. (2003). Relationship between age of recognition of first disturbances and severity in young children with autism. European Child \& Adolescent Psychiatry, 12(3), 122-127.

Barbaro, J. (2009). Autism Spectrum Disorders in infancy and toddlerhood: A review of the evidence on early signs, early identification tool, and early diagnosis. Journal of Developmental \& Behavioral Pediatrics, 30(5), 447-459.

Bardin, L. (1977). Análise de Conteúdo. Lisboa, Portugal: Edições 70.

Becker, M. M., Wagner, M. B., Bosa, C. A., Schmidt, C., Longo, D., Papaleo, C., \& Riesgo, R. S. (2012). Translation and validation of Autism Diagnostic Interview-Revised (ADI-R) for autism diagnosis in Brazil. Arquivos de Neuropsiquiatria,70(3), 185-190.

Bosa, C. (2002a). Atenção compartilhada e identificação precoce do autismo. Psicologia, Reflexão e Crítica, 15(1), 77-88.

Bosa, C. (2002b). Sinais precoces de comprometimento social no autismo infantil. In W. Camargos, Jr. W. (Ed.), Transtornos invasivos do desenvolvimento (pp. 42-47). Brasília: Corde.

Bosa, C. (2009). Compreendendo a evolução da comunicação do bebê: implicações para a identificação precoce do autismo. In V. G. Haase, F. O. Ferreira, \& F. J. Penna (Eds.), Aspectos biopsicossociais da saúde na infância e adolescência (pp. 319-328). Belo Horizonte: Coopmed.

Castro-Souza, R. M., \& Pasquali, L. (2011). Adaptação brasileira do M-CHAT (Modified Checklist for autism in toddlers). Dissertação de mestrado não publicada, Universidade de Brasília, Brasília.

Chakrabariti, S. (2009). Early identification of Autism. Indian Pediatrics, 46(17), 412-414.

Charman, T., Swerrwnham, J., Baron-Cohen, S., Cox, A., Braid, G., \& Drew, A. (1997). Infants with autism: an investigation of empathy, pretend play, joint attention, and imitation, Developmental Psychology, 33(5), 781-789.

Chawarska, K., Paul, R., Klin, A., Hannigen, S., Dichtel, L., \&Volkmar, F. (2007) Parental recognition of developmental problems in toddlers with ASD. Journal of Autism and Developmental Disorder, 37, 62-73.
Coonrod, E. E., \& Stone, L. L. (2004).Early concerns of parents of children with autistic and nonautistic disorders. Infants and Young Children, 17(3), 258-268.

Daley, T. (2004). From symptom recognition to diagnosis: children with autism in urban India. Social Science \& Medicine, 58, 1323-1335.

De Giacomo, A., \& Fombonne, E. (1998). Parental recognition of developmental abnormalities in autism. European Child \& Adolescent Psychiatry, 7(3), 131-136.

Elsabbagh, M., Divan, G., Koh, Y., Kim, Y. S., Kauchali, S., Marcín, C., ... Fombonne, E. (2012). Global prevalence of autism and other pervasive developmental disorders. Autism Research, 5(3), 160-179.

Fombonne, E. (2009). Epidemiology of pervasive developmental disorders. Pediatric Research, 65(6), 591-598.

Gray, K., \& Tonge, B. (2001). Are there early features in autism in infants and preschool children? Journal of Paediatrics and Child Health, 37, 221-226.

Howlin, P., \& Asgharian, A. (1999). The diagnosis of autism and Asperger syndrome: Findings from a survey of 770 families. Developmental Medicine and Child Neurology, 41, 834-839.

Howlin, P., Magiati, I., \& Charman, T. (2009). Systematic review of early intensive behavioral interventions for children with autism. American Journal of Intellectual Development Disabilities, 37, 23-41.

Hus, V., Taylor, A., \& Lord, C. (2011). Telescoping of caregiver report on the Autism Diagnostic Interview-revised. Journal of Child Psychology and Psychiatry, 52(7), 753-760.

Järbrink, K., \& Knapp, M. (2001). The economic impact of autism in Britain.Autism, 5(1), 7-22.

Johnson, C. P. (2008). Recognition of autism before age 2 years. Pediatrics in Review, 29, 86-96.

Lord, C., Storoschuk, S., Rutter, M., \& Pickles, A. (1993). Using the ADI-R to diagnose autism in preschool children. Infant Mental Health Journal, 14(3), 234-251.

Maestro, S., Muratori, F., Cavallaro, C., Pei, F., Stern, D., Golse, B., \& Palacio-Espasa, F. (2002). Attentional skills during the first 6 months of age in autism spectrum disorder. Journal of the American Academy of Child and Adolescent Psychiatry, 41, 1239-1245.

Mandell, D. S., Listerud, J., Levy, S. E., \& Pinto-Martin, J. A. (2002). Race differences in the age among medicaid-eligible children with autism. Journal of American Academy of Child and Adolescent Psychiatry, 41, 1447-1453.

Mandell, D. S., Wiggins, L. D., Carpenter, L. A., Daniels, J., Di Guiseppi, C., Durkin, M. S., ... Kirby, R. S. (2009). Racial/ ethnic disparities in the identification of children with autism spectrum disorders. American Journal of Public Health, 99, 493-498.

Mandell, D., Novak, M., \& Zubritsky, C. (2005). Factors associated with age of diagnosis among children with autism spectrum disorders. Pediatrics, 116, 1480-1486.

Marques, D. F. \& Bosa, C. A. (2010). Aprimoramento e evidências de validade do protocolo de observação para crianças com suspeita de transtorno do espectro autista: um estudo preliminar. Dissertação de mestrado não publicada,Universidade Federal do Rio Grande do Sul, Porto Alegre. 
Montenegro, M. N. \& Mercadante, M. T. (2007). Avaliação e estudo dos comportamentos de orientação social e atenção compartilhada nos transtornos invasivos do desenvolvimento. Dissertação de mestrado não publicada, Universidade Presbiteriana Mackenzie.

Naber, F., Bakermans-Kranenburg, M. J., Van Ijzendoorn, M. H., Dietz, C., Van Daalen, M., Swinkels, S. H., ... Engeland, H. (2008). Joint attention development in toddlers with autism. European Child \& Adolescent Psychiatry, 17, 143-152.

Noterdaeme, M., \& Hutzelmeyer-Nickels, A. (2010). Early symptoms and recognition of pervasive developmental disorders in Germany. Autism, 14(6), 575-588.

Ozonoff, S., Iosif, A. M., Baguio, F., Cook, I. C., Hill, M. M., Hutman, T., ... Young, G. S. (2010). A prospective study of the emergence of early behavioral signs of autism. Journal of the Academy of Child \& Adolescent Psychiatry, 49(3), 256-66.

Papaeliou, C. F., \& Trevarthen, C. (2006). Prelinguistic pitch patterns expressing 'communication' and 'apprehension'. Journal of Child Language, 33, 163-178

Pickles, A., Simonoff, E., Conti-Ramsden, G., Falcaro, M., Simkin, Z., Chandler, S., ... Baird, G. (2009). Loss of language in early development of autism and specific language impairment. Journal of Child Psychology and Psychiatry, 50(7), 843-852.

Reichow, B. (2011). Overview of meta-analyses on early intensive behavioral intervention for young children with autism spectrum disorders. Journal of Autism and Development Disorders, 42(4), 512-520.

Rice, C. E., Baio, J., Van Naarden, K., Doernberg, N., Meaney, F. J., \& Kirby, R. S. (2007). A public health collaboration for the surveillance of autism spectrum disorders. Paediatric and Perinatal Epidemiology, 21(2), 179-190.

Robson, C. (1995). Real Word Research. Oxford: Blackwell.

Rutter, M. L. (2011). Progress in understanding autism: 2007-2010. Journal of Autism and Developmental Disorders, 41, 395-404.

Saint-Georges, C., Cassel, R., Cohen, D., Chetouani, M., Laznik, M., Maestro, S., \& Muratoni, F. (2010). What studies of family home movie can teach us about autistic infants: a literature review. Research in Autism Spectrum Disorders, 4, 355-366.

Shattuck, P. T., Durkin, M., Maenner, M. B. S., Newschaffer, C., Mandell, D. S., Wiggins, L., ... Cuniff, C. (2009). Timing of identification among children with an autism spectrum disorder: Findings from a population-based surveillance study. Journal of American Academy of Child and Adolescent Psychiatry, 48(5), 474-483.

Shumway, S., \& Wetherby, A. (2009). Communicative acts of children with autism spectrum disorders in the second year of life. Journal of Speech, Language, and Hearing Research, 52, 1139-1156.

Sifuentes, M. S., \& Bosa, C. A. (2007). A coparentalidade em pais de crianças com autismo em idade pré-escolar. Dissertação de mestrado não publicada, Universidade Federal do Rio Grande do Sul, Porto Alegre.

Siklos, S., \& Kerns, K. (2007). Assessing the diagnostic experiences of a small sample of parents of children with autism spectrum disorders. Research in Developmental Disabilities, 28, 9-22.

Sudman, S., \& Bradburn, N. M. (1973). Effects of time and memory factors on response in surveys. Journal of the American Statistical Association, 68, 805-815.
Tomasello, M. (2003). Origens culturais da aquisição do conhecimento humano. (C. Berliner, Trans.). São Paulo: Martins Fontes (Trabalho original publicado em 1999)

Turner, M. (1999). Annotation: repetitive behaviour in autism: a review of psychological research. Journal of Child Psychology and Psychiatry, 40(6), 839-849.

Watt, N., Wetherby, A. M., Barber, A., \& Morgan, L. (2008). Repetitive and stereotyped behaviors in children with autism spectrum disorders in the second year of life. Journal of Autism and Developmental Disorders, 38,1518-1533.

Werner, E. \& Dowson, G. (2005).Validation of the phenomenon of autistic regression using home videotapes. Archives of General Psychiatry, 62, 889-895.

Werner, E., Dawson, G., Munson, J., \& Osterling, J. (2005).Variation in early developmental course in autism and its relation with behavioral outcome at 3-4 years of age. Journal of Autism and Developmental Disorders, 35(3), 337-350.

Wiggins, L. D., Baio, J., \& Rice, C. (2006). Examination of the time between first evaluation and first autism spectrum diagnosis in a population-based sample. Developmental and Behavioral Pediatrics, 27(2), 79-87.

Wiggins, L. D., Rice, C. E., \& Baio, J. (2009).Developmental regression in children with an autism spectrum disorder identified by a population-based surveillance system. Autism, 13(4), 357-374.

Yeargin-Allsopp, M., Rice, C., Karapurkar, T., Doernberg, N., Boyle, C., \& Murphy, C. (2003). Prevalence of autism in a US metropolitan area. The Journal of the American Medical Association, 289(1), 49-55.

Yoder, P., Stone, W. L., Walden, T., \& Malesa E. (2009). Predicting social impairment and ASD diagnosis in younger siblings of children with autism spectrum disorder. Journal of Autism and Developmental Disorders, 39(10), 1381-1391.

Zwaigenbaum, L., Bryson, S., Rogers, T., Roberts, W., Brian, J., \& Szatmari, P. (2005). Behavioral manifestations of autism in the first year of life. International Journal of Developmental Neuroscience, 23(2-3), 143-152.

Zwaigenbaum, L., Bryson, S., Lord, C., Rogers, S. Carter, A., Carver, L., ... Yirmiya, N. (2009). Clinical assessment and management of toddlers with suspected autism spectrum disorder: Insights from studies of high-risk infants. Pediatrics, 123(5), 1383-1391. 